\title{
Inserção da análise do comportamento na educação: o estado do conhecimento de teses e dissertações produzidas entre 2010 e 2015
}

\begin{abstract}
Resumo: No contexto educacional formal, a análise do comportamento estuda as relações que ocorrem entre sujeitos dentro das salas de aula. Prática pedagógica, ou ensinar, é definida como planejar situações que favoreçam a aprendizagem dos alunos. O presente trabalho é uma pesquisa bibliográfica do tipo estado do conhecimento. O objetivo é verificar em teses e dissertações produzidas entre 2010 e 2015 como conceitos da Análise do Comportamento foram aplicados em contextos educacionais A coleta de dados foi feita no Portal Brasileiro de Publicações Científicas em Acesso Aberto, a partir das seguintes palavras-chaves: I) Análise do Comportamento; II) Behaviorismo Radical; III) B. F. Skinner; IV) Educação Infantil; V) Ensino Fundamental; VI) Ensino Médio; VII) Ensino Superior; VIII) Educação de Jovens e Adultos; IX) Educação Profissional e Tecnológica; X) Educação Indígena; XI) Educação Especial; XII) Educação do Campo; e XIII) Educação Ambiental. As palavras-chaves foram divididas em dois grupos: a) Análise do Comportamento (I, II e III) e b) níveis e modalidades de ensino (de IV a XIII). Cada item do primeiro grupo foi combinado com cada item do segundo grupo. As buscas encontraram 35 trabalhos (15 teses e 20 dissertações), dos quais 77,14\% são oriundos da região Sudeste. Pontifícia Universidade Católica de São Paulo e Universidade Federal de São Carlos produziram 51,43\% dos trabalhos selecionados. Em relação às práticas pedagógicas, o conceito mais frequente foi matching-to-sample, que apareceu em 18 destes. Em relação aos níveis de ensino, o ensino fundamental obteve a maior concentração de trabalhos: 34,29\%. Sobre as modalidades de ensino, somente a Educação Especial foi citada.
\end{abstract}

Palavras-chave: Behaviorismo. Análise do comportamento. Educação.

\section{Introdução}

A Análise do Comportamento, em sua perspectiva radical, é uma ciência embasada nos estudos do cientista e filósofo norte americano B. F. Skinner (1904 - 1990). Seu objeto de estudo é o comportamento, que é definido como relação entre um organismo, seja ele humano ou não humano, e o ambiente. Divide-se em três principais eixos: I) Análise Experimental do Comportamento - que estuda princípios básicos do comportamento, II) Behaviorismo Radical - filosofia que norteia os campos de prática e pesquisa da Análise do Comportamento e III) Análise do Comportamento Aplicada - aplicação dos conhecimentos dos outros dois eixos no cotidiano. (SKINNER, 2006; TOURINHO, 1999)
Fernando Nóbrega Universidade Federal da Bahia fernando.nobrega@live.com

Paulo Roberto Holanda Gurgel Universidade Federal da Bahia pgpaulogurgel@gmail.com 
(1) O objetivo, aqui, é deixar claro o que está sendo denominado por prática pedagógica. Discutir possíveis divergências sobre a definição desse termo entre os autores citados não é um objetivo do presente artigo.
Uma das áreas de interesse de Skinner foi a Educação (SKINNER, 1972), para a qual ele prescreveu, embasado no conhecimento da Análise Experimental do Comportamento sobre princípios de aprendizagem, uma série de mudanças que poderiam melhorar a qualidade da educação formal. De acordo com Skinner (2003), uma comunidade que ensina comportamentos úteis, no sentido de facilitar a resolução de problemas futuros-individuais e/ou coletivos-, aumenta suas chances de sobrevivência. Entretanto, Skinner afirma que a Educação é falha no propósito de ensinar comportamentos úteis, o que o deixou preocupado quando se trata dasprováveis consequências indesejáveis derivadas dessa fragilidade.

Em uma visão skinneriana, ensinar é programar o ambiente do aluno de tal forma que a aprendizagem seja favorecida. (SKINNER, 1972) Programar um ambiente sem o conhecimento técnico adequado de relações entre sujeitos e ambiente parece ser uma tarefa difícil, já que, tradicionalmente, negligencia-se tais relações. (SKINNER, 2006; 2007) Moroz (1993) afirma que a aprendizagem é função da relação entre aluno e professor, em trabalho colaborativo.

Denominamos de prática pedagógica o comportamento do professor de facilitar a aprendizagem do aluno. Lobato (2001) afirma que uma prática pedagógica precisa ser: I) intencional, II) sistematizada e III) planejada. De forma semelhante a Lobato (2001), Saviani (2005) define prática pedagógica como sendo a organização e realização do ato educativo. Carvalho (2015, p. 44), por sua vez, define o termo como "fazer educativo [...] ou prática educativa". ${ }^{1}$

Apesar de não utilizar o termo prática pedagógica, Skinner (1972, p. 4) aponta que o fazer do professor diz respeito ao planejamento do ambiente de sala de aula:

\footnotetext{
Tanto quanto aqui nos ocupa, ensinar é simplesmente arranjar contingências de reforço. Entregue a si mesmo, em dado ambiente, um estudante aprenderá, mas nem por isso terá sido ensinado. A escola da vida não é bem uma escola, não por que ninguém nela aprende, mas por que ninguém ensina. Ensinar é o ato de facilitar a aprendizagem; quem é ensinado aprende mais rapidamente do que quem não é.
}

O que, na citação acima, Skinner (1972) chama de contingências de reforço é um conceito importante na Análise do Comportamento e que está intimamente ligado ao conceito de comportamento 
operante, que é aquele sensível às consequências que produz. (CATANIA, 1999) Uma contingência é uma relação de dependência entre: I) as respostas emitidas pelo organismo; II) o contexto em que elas são emitidas; e III) as consequências que as respostas produzem. (CATANIA, 1999) Uma contingência de reforço é uma relação de dependência entre contexto, respostas e consequências reforçadoras, que são aquelas que mantém ou aumentam a taxa de emissão das respostas que as produziram. (CATANIA, 1999; SKINNER, 2003) Dessa maneira, pode-se afirmar que consequências reforçadoras são importantes para o estabelecimento de novos comportamentos.

Da mesma forma que uma consequência reforçadora tem efeito sobre a resposta que a produziu, tem também efeitos em relação ao contexto sobre o qual a resposta foi emitida: um estímulo correlacionado com o reforçamento de uma resposta operante recebe o nome de estímulo discriminativo e possui efeito evocativo desta resposta reforçada no passado. (CATANIA, 1999) Por exemplo: uma criança pediu algumas vezes sorvete para a mãe pouco antes do almoço e ouviu um não como resposta. Por outro lado, essa criança pediu sorvete algumas vezes para a avó, que atendeu seu pedido. A avó funciona, provavelmente, como um estímulo discriminativo para o comportamento operante de pedir sorvete. Por outro lado, a mãe funciona provavelmente como um estímulo delta - o tipo de estímulo correlacionado com o não reforçamento de operantes. Dessa maneira, é mais provável que o pedido seja feito para a avó e não para a mãe.

Existem ocasiões em que um dado estímulo só possui função de estímulo discriminativo se houver um outro estímulo presente. Sério e colaboradores (2010) trazem o exemplo de Victor de Aveyron para ilustrar esse tipo de ocasião. Victor, uma criança que vivia só na natureza, aproximou-se da localidade Saint Sernin no inverno de 1800. Victor chamou a atenção de profissionais de diversas áreas do conhecimento e foi encaminhado para Paris, onde foi apresentado ao médico Jean Marc Gaspard Itard, que defendia a possibilidade de educá-lo. Depois de algum tempo de treino, Itard conseguiu que Victor formasse a palavra LAIT (leite, em francês). Ele dispôs para o menino um quadro com contornos das letras desenhadas e as próprias letras moldadas em metal - a letra L deveria ser colocada no contorno em forma de L e a letra A deveria ser colocada no contorno em forma de A - o mesmo se aplicava para as letras I e 
T. A consequência reforçadora para esse operante - organizar as letras - era uma xícara de leite. Sério e colaboradores (2010, p. 92) afirmam que "[...] as consequências diferenciais para o responder diante de determinado estímulo dependiam da relação entre esse estímulo [...] e um outro estímulo". Para produzir a consequência reforçadora "xícara de leite", Victor deveria organizar as letras na ordem correta e colocá-las dentro de seus respectivos contornos. Sobre essa relação entre estímulos antecedentes, Catania (1999, p. 396) afirma que uma discriminação condicional é aquela "em que o reforço do responder na presença de um estímulo depende de (é condicional a) outros estímulos". O processo envolvido com a discriminação condicional é chamado de matching-to-sample (MTS) - emparelhamento com o modelo. (SKINNER, 2005 [1950])

Existem situações em que o comportamento de outras pessoas serve como estímulo discriminativo para o comportamento de imitar. (CATANIA, 1999) Bandura (1962, p. 215) defende que imitar é "emitir comportamentos ou atitudes similares àqueles exibidos por modelos reais ou simbólicos". Imitar, como um tipo de operante, é sensível às consequências que produz - o que significa que, caso produza consequências reforçadoras, terá sua probabilidade de ocorrência futura aumentada. Caso o comportamento de imitar esteja estabelecido, novos comportamentos podem ser aprendidos de forma mais eficiente e rápida pela imitação do que por outros meios. (CATANIA, 1999) Um fenômeno relacionado com a imitação é a modelação, que é o processo de aquisição de novos comportamentos a partir de modelos. (BANDURA, 1962) Bandura (1965) realizou um experimento sobre comportamentos agressivos, que contou com 36 crianças do sexo masculino e 36 do sexo feminino. Desse total, 48 crianças foram expostas a modelos e 24, o grupo controle, não. O experimento consistiu em expor um grupo de crianças a um modelo que estava emitindo respostas agressivas durante uma brincadeira - chutar, empurrar e/ou bater no brinquedo enquanto que o outro grupo foi exposto a um modelo que estava emitindo outros tipos de respostas (não agressivas). Após a exposição, as crianças foram colocadas em uma sala experimental com brinquedos e foram observadas através de um espelho unidirecional. As crianças que foram expostas ao modelo de respostas agressivas, apresentaram esse tipo de responder. As crianças que foram expostas ao modelo de respostas 
não agressivas emitiram comportamentos agressivos em menor frequência que o grupo controle.

Outra maneira de ensinar comportamentos é através do conceito de regra, que está intimamente ligado ao conceito de comportamento verbal. Sério e colaboradores (2010, p. 138) definem comportamento verbal da seguinte forma: "[...] comportamento verbal é comportamento operante que é reforçado pela mediação de outras pessoas que foram especialmente preparadas para reagir como mediadores". Baum (2006) define regra como sendo a descrição verbal (mesmo que incompleta) de uma contingência. Quando um professor de matemática mostra algum "pulo do gato" para resolver um problema complicado, ele está fornecendo uma regra - "usem esse artificio e vocês conseguirão resolver esse problema". Quando um professor de natação manda seu aluno fazer o movimento de "S" com a mão embaixo da água, ele também está fornecendo uma regra - "emite esse comportamento e você chegará mais rápido e com menos esforço na outra borda da piscina". Nos dois exemplos, as regras fornecidas versam sobre comportamentos que, se emitidos, produzirão consequências reforçadoras - o problema matemático será resolvido e o deslocamento na água será feito de forma mais eficiente.

Um professor pode, ainda, ensinar novos comportamentos de acordo com as consequências diferenciais que ele dispõe para os comportamentos de seus alunos. Quando algumas respostas são reforçadas em detrimento de outras, está ocorrendo o reforçamento diferencial. A modelagem é uma maneira de ensinar comportamentos através do reforçamento diferencial, que é feito com base em critérios de aproximação de um comportamento alvo - o comportamento que o aluno precisa aprender. (CATANIA, 1999) Um exemplo tradicional da Psicologia diz respeito ao experimento realizado na caixa de Skinner, em que um sujeito experimental (rato de laboratório) é colocado em uma caixa com uma barra que, se pressionada, acionará um bebedouro que, por sua vez, liberará uma gota de água. Respostas de I) aproximar-se, II) erguer-se próximo à barra, III) ultrapassar a altura da barra, IV) colocar uma pata dianteira sobre a barra, V) colocar as duas patas dianteirassobre a barra e VI) pressionar a barra são reforçadas de forma diferencial - I é reforçada algumas vezes e, depois, não mais. É provável que II ocorra enquanto I não é reforçada (e a partir desse momento, somente II é reforçada). Quando II não for mais 
reforçada, é provável que III ocorra (e, se assim for, será reforçada). O comportamento final é o de pressionar a barra.

Já que "ensinar é simplesmente arranjar contingências de reforço" (SKINNER, 1972, p. 4), uma contribuição analítica comportamental à Educação diz respeito ao planejamento prévio e ao estabelecimento de contingências de reforço, para, assim, favorecer a aprendizagem dos alunos.

Mesmo preocupado com a Educação, Skinner é negligenciado tanto do ponto de vista teórico quanto do ponto vista prático na Educação brasileira. De acordo com Gioia (2001), a Análise do Comportamento é ensinada de forma equivocada para professores, que, ao conhecerem uma teoria que supostamente busca "adestrar" alunos através de punições e recompensas, afastam-se dela; o que diminui drasticamente as chances de a Análise do Comportamento embasar suas práticas pedagógicas. Concordando com Gioia (2001), Henklain e Carmo (2013, p. 706), chegamos à seguinte afirmação:

\footnotetext{
Consideramos que essa abordagem tem contribuições importantes para oferecer aos educadores. Contudo, a insistência em classificar a abordagem comportamental à educação como tecnicista e redutora do homem, a despeito de diversos trabalhos que demonstraram o contrário (CARMO; BAPTISTA, 2003; CARRARA, 2005; LUNA, 2000; TEIXEIRA, 2006; TODOROV; MOREIRA, 2008; RODRIGUES, 2011; SKINNER, 1969, 1972 [1968], 1991 [1989]), cria barreiras a um diálogo proveitoso e ao desenvolvimento e implementação de propostas viáveis que podem colaborar com os diferentes esforços empreendidos para ajudar a educação brasileira a melhorar.
}

Conhecimentos imprecisos não são um fenômeno exclusivo da atualidade. (PACCA, 2013) Skinner (2006) listou e analisou as vinte críticas mais comuns feitas ao Behaviorismo Radical naquela época e concluiu que todas são equivocadas, elencando algumas possíveis explicações para tamanha resistência ao ponto de vista behaviorista radical, como, por exemplo: I) a não diferenciação entre os behaviorismos de Watson - o metodológico - e de Skinner - o radical; e II) paradigmas explicativos alternativos existem a tempo suficiente para que sejam, de certa forma, compreendidos pelo senso comum, os quais os adota como verdade.

Dado o amplo contexto de oposição, uma pesquisa sobre o que já foi produzido sobre a inserção da Análise do Comportamento na Educação é importante para sistematizar as produções acadêmicas 
e os possíveis avanços na área, bem como possíveis lacunas teórico-práticas, além de evidenciar a aplicabilidade dos princípios analítico-comportamentais em contexto educacional. Assim sendo, o objetivo do presente artigo é verificar em teses e dissertações produzidas entre 2010 e 2015 como conceitos da Análise do Comportamento foram aplicados em contextos educacionais.

\section{Método}

O presente artigo é uma pesquisa bibliográfica do tipo estado do conhecimento. O corpus analisado constitui-se de teses e dissertações pesquisadas através do Portal Brasileiro de Publicações Científicas em Acesso Aberto ${ }^{2}$ As palavras-chaves podem ser divididas em dois grupos: o primeiro grupo diz respeito à Análise do Comportamento e possui os itens I) Análise do Comportamento, II) Behaviorismo Radical e III) B. F. Skinner. O segundo grupo diz respeito aos Níveis e às Modalidades de ensino, de acordo com a Lei de Diretrizes e Bases (LDB) e possui os itens I) Educação Infantil, II) Ensino Médio, III) Ensino Superior, IV) Educação de Jovens e Adultos, V) Educação Profissional e Tecnológica, VI) Educação Indígena, VII) Educação à Distância, VIII) Educação Especial, IX) Educação do Campo e X) Educação Ambiental. Cada um dos itens do primeiro grupo - ou seja, Análise do Comportamento -, foi combinado com cada um do segundo grupo - Níveis e Modalidades de ensino -, fazendo com que, dessa forma, os resultados da pesquisa sejam mais amplos.

Como a pesquisa foi feita com duas palavras-chaves, um refinamento a priori precisou ser feito. Na página inicial do portal, foi selecionado o link Busca Avançada, que dá a possibilidade de pesquisar com mais de uma palavra-chave. Nessa página, foi selecionado também o recorte temporal da pesquisa, que é entre os anos de 2010 e 2015. Este período foi escolhido por permitir a seleção de trabalhos atuais e por ser suficiente para conhecer as tendências na produção acadêmica em pós-graduações stricto sensu na temática da inserção da Análise do Comportamento na Educação. ${ }^{3}$

Este período foi escolhido por: I) ter sido suficiente para conhecer as tendências na produção em pós-graduações stricto sensu e II) de forma que os trabalhos selecionados foram considerados atuais. Pode, então, ser feita a pesquisa de fato, mas refinamentos
(2) Disponível em: < http://oasisbr. ibict.br/vufind/>.

(3) A afirmação de que selecionar trabalhos produzidos entre 2010 e 2015 permite conhecer as

tendências na produção acadêmica em pós-graduações stricto sensu na temática da inserção da Análise do Comportamento na Educação é embasada em pesquisas preliminares, em que se percebeu que os trabalhos produzidos nesta temática, no século XXI, concentram-se neste intervalo de tempo. 
(4) Esse critério foi criado com base em pesquisas preliminares, através das quais se percebeu que existiam resultados em que 0 termo Análise do Comportamento possui somente similaridade topográfica com o nome da ciência do comportamento. Por exemplo: análise do comportamento das moléculas do aço em situações de calor extremo. a posteriori precisaram ocorrer (seleção de opções na coluna à esquerda da página): I) seleção do idioma Português e II) seleção de documentos Open Acess (de acesso aberto).

Após os refinamentos feitos, todos os trabalhos listados foram selecionados através de exame dos títulos e resumos. Os critérios de inclusão foram: I) versar sobre a Análise do Comportamento tendo como autor referência B.F. Skinner ${ }^{4}$ e II) versar sobre alguma prática pedagógica. Após essa seleção prévia, foi criada uma planilha, através do aplicativo Microsoft Excel para abarcar os seguintes dados: I) Título do trabalho, II) Tipo de trabalho (tese ou dissertação), III) Autor, V) Ano de publicação, VI) Região do país, VIII) Instituição; X) Nível de ensino e XI) Modalidade de ensino.

\section{Resultados e discussão}

Na presente seção, será realizada a apresentação dos dados agrupados da seguinte forma: 3.1) Distribuição geográfica e institucional dos relatórios e 3.2) Conceitos que embasam as práticas e público alvo.

\section{Distribuição geográfica e institucional dos relatórios}

Com base nos critérios supracitados, foram selecionados35 trabalhos, sendo 15 teses e 20 dissertações. Pode-se observar a ausência de publicações nas regiões norte e nordeste. A Figura 1, a seguir, ajuda a perceber que a maior parte dos relatórios publicados são oriundos da região Sudeste (27 trabalhos), seguida pela região Sul (7 trabalhos) e, por último, a região Centro-Oeste (1 trabalhos).

Figura 1

Regiões de origem das publicações

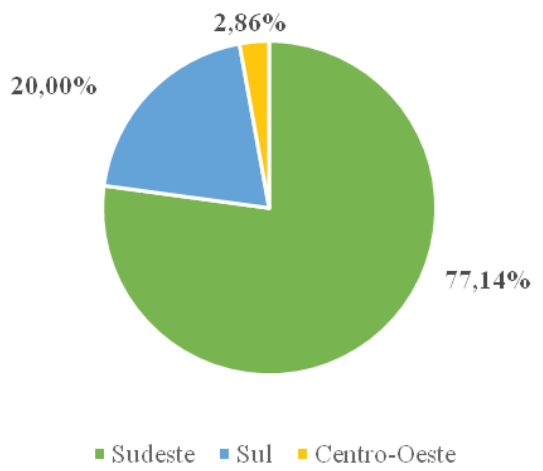


$\mathrm{Na}$ região Sudeste, foram encontradas algumas das instituições com tradição no campo quando se fala em Análise do Comportamento, como, por exemplo, a Pontifícia Universidade Católica de São Paulo (PUC-SP) e a Universidade Federal de São Carlos (UFSCar). Tal fato parece estar em harmonia com o constatado na presente pesquisa: a região Sudeste foi a que mais produziu relatórios de pesquisa que aplicaram os conhecimentos analítico-comportamentais em contextos educacionais, o que corrobora a afirmação de Barros (2000), que aponta que as regiões Sul e Sudeste são polos de produção científica no país. Possivelmente as desigualdades sociais observadas entre as regiões do Brasil ocorrem de forma concomitante com desigualdades do ponto de vista de investimentos em pesquisas científicas. Instituições que recebem mais investimentos, públicos e/ou privados, tendem a desenvolver mais conhecimento científico, atraindo, inclusive, mais pesquisadores. Essa tendência pode ajudar a explicar o fato de que, no ano 2000, mais de $70 \%$ de mestres e $91 \%$ dos doutores recém titulados encontravam-se na região Sudeste. (BARROS, 2000) É possível que, em um intervalo de tempo de dezessete anos entre as afirmações de Barros (2000) e a presente pesquisa, não tenham ocorrido mudanças significativas nessa realidade, já a região Sudeste foi a maior produtora de relatórios sobre Análise do Comportamento e Educação.

A seguir, na Figura 2, é possível observar a frequência relativa de publicações das instituições da região Sudeste:

Figura 2

Frequência relativa de publicações por Instituição

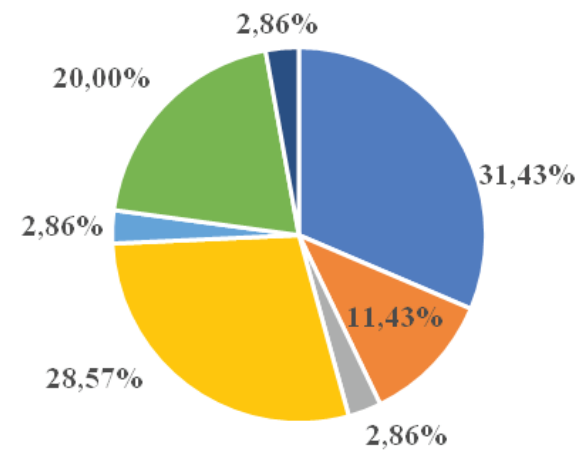

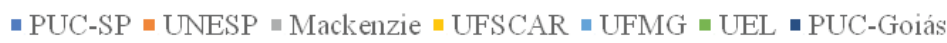


As instituições PUC-SP e UFSCar publicaram, respectivamente, 11 e 10 trabalhos. Em terceiro lugar, ficou a Universidade Estadual Paulista (UNESP), com 4 publicações, seguida pela Universidade Presbiteriana Mackenzie, com uma publicação. A Universidade Federal de Minas Gerais (UFMG), também com uma publicação, foi a única instituição do Sudeste do Brasil não pertencente ao estado de São Paulo que apresentou produção. De acordo com o gráfico acima, pode-se notar que o estado de São Paulo é o que mais produziu relatórios nessa temática.

Quando a comparação entre instituições envolve todas as que apresentaram relatórios selecionados, pode-se afirmar que há considerável diferença entre a taxa de publicações da PUC-SP, da UFSCar e da Universidade Estadual de Londrina (UEL) em relação às outras instituições: essas três universidades, juntas, são responsáveis por $80 \%$ dos trabalhos selecionados.

Em relação aos anos das publicações, observa-se, de 2011 a 2013, um aumento no número de trabalhos; mas, em 2014 e 2015, ocorre um declínio na taxa de produções. A Figura 3 ajuda a ilustrar a dispersão da produção no intervalo de tempo selecionado.

Figura 3

Quantidade de publicações em cada ano do recorte temporal

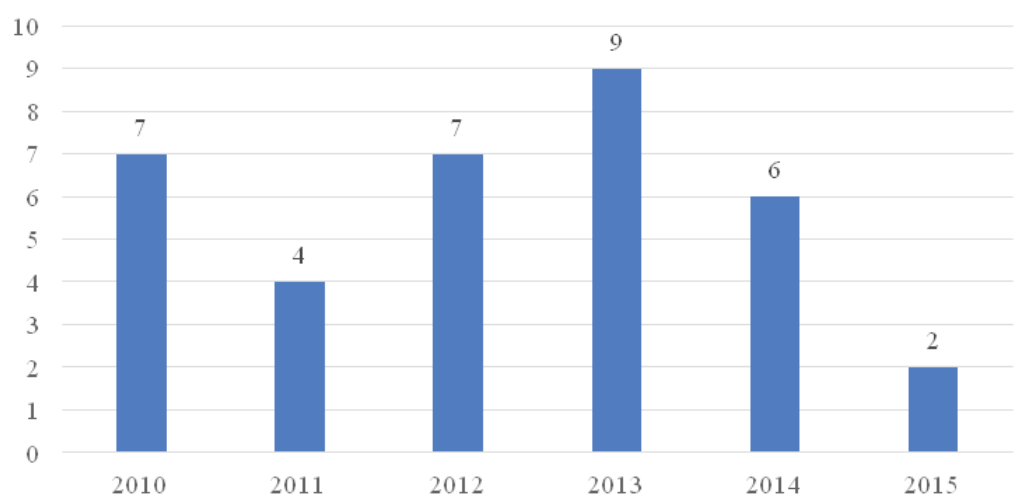

Conceitos que embasam as práticas e público alvo

A maior parte dos trabalhos analisados explicita a prática pedagógica utilizada - somente um trabalho não o fez, referindose às práticas como "condições de ensino planejadas". O conceito matching-to-sample é o mais referido como base para as práticas relatadas, aparecendo em 51,43\% dos relatórios, seguido pelo termo 
regras, que aparece em 34,29\% dos trabalhos. A Figura 4, a seguir, permite visualizar a frequência absoluta de ocorrência dos termos que embasam as práticas pedagógicas. Como existem trabalhos que utilizam mais de um termo, regras e modelação, por exemplo, optou-se por utilizar, como medida, a frequência absoluta.

Figura 4

Frequência absoluta de termos que embasam as práticas pedagógicas

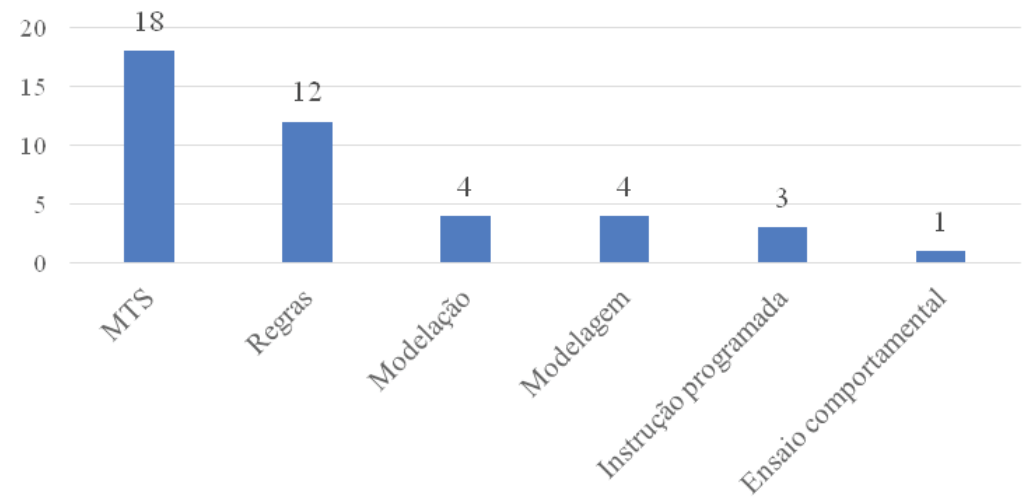

Ao analisar o nível de ensino em que ocorreram as pesquisas estudadas, percebe-se que a maior parte (34,29\%) ocorreu no ensino fundamental, enquanto que $20 \%$ ocorreu no ensino superior. Pesquisas realizadas com alunos de ensino médio e de educação infantil representam, respectivamente, 8,57\% e 5,71\% da amostra. Em relação às modalidades de ensino, somente a educação especial foi referida (por 17,14\% dos trabalhos). Tal dado sugere que pesquisadores da Análise do Comportamento talvez não se debrucem sobre diversos públicos, como, por exemplo, índios, quilombolas, indivíduos que realizam cursos técnicos e cursos relacionados à educação de jovens e adultos. Dado que a Análise do Comportamento não é uma teoria amplamente difundida no campo educacional, diversificar o público alvo de intervenções parece ser uma estratégia interessante, uma vez que isso aumentaria a probabilidade de educadores não behavioristas conhecerem a teoria e alguns procedimentos analítico-comportamentais. Um dado que pode ser compreendido como um exemplo dessa diversificação diz respeito ao fato de que $8,57 \%$ das pesquisas constituíram-se como treino de pais (comportamentos dos pais que podem ajudar na educação dos filhos que foram ensinados) e de professores 
(comportamentos no contexto sala de aula que podem potencializar o aprendizado dos alunos).

Quanto aos resultados expostos, percebe-se que pouco mais da metade dos relatórios (51,43\%) foram considerados adequados - as intervenções obtiveram êxito. Em 31,43\% dos trabalhos, entretanto, os resultados foram considerados como a melhorar - as mudanças comportamentais obtidas foram inexpressivas. Outros trabalhos, 8,57\%, não puderam ter seus resultados categorizados dessa maneira binária, como é o caso do de Jardim (2014), que investigou qual método seria mais eficiente na tarefa de ensinar comportamentos deficitários para crianças diagnosticadas com síndrome de Down: se estória social - uma maneira de ensinar comportamentos através de regras -, se reforçamento diferencial ou se a combinação dessas duas intervenções.

\section{Considerações finais}

A forma como a pesquisa ocorreu permitiu que um panorama da produção stricto sensu brasileira sobre a inserção da Análise do Comportamento na Educação no período entre os anos 2010 e 2015 fosse apreciado. Dessa forma, algumas características da produção acadêmica sobre esse tema puderam ser explicitadas, como, por exemplo, o destaque da região Sudeste na publicação de relatórios de pesquisa sobre esse tipo de conhecimento, a prevalência do matching-to-sample como procedimento em mais da metade dos trabalhos e a baixa frequência da instrução programada enquanto processo de ensino.

O presente trabalho, entretanto, provavelmente não abarca a totalidade da produção stricto sensu sobre o tema. Novas buscas, com diferentes combinações de palavras-chaves, possivelmente alcançariam resultados diferentes. Hipotetiza-se, então, que, mesmo mantendo o recorte temporal adotado nesse trabalho, novas buscas, com outras palavras-chaves, podem complementar os resultados aqui apresentados, resultando em um panorama mais completo, que pode contribuir com uma maior inserção da Análise do Comportamento na Educação, porque identifica lacunas teóricopráticas e explicita que conceitos analítico-comportamentais embasam as práticas pedagógicas utilizadas pelos pesquisadores. 


\title{
Insertion of behavior analysis in education: the state of knowledge of doctoral thesis and master dissertations produced between 2010 and 2015
}

\begin{abstract}
In the formal educational context, Behavior Analysis studies the relations that occur between subjects in the classrooms. Pedagogical practice, or teaching, is defined as planning situations that favor student learning. The present study is a state of knowledge bibliographic research. The objective is to verify in doctoral thesis and master dissertations produced between 2010 and 2015 how concepts of Behavior Analysis were applied in educational contexts. Oncethe data were collected in the Brazilian Scientific Publishing Portalthe key word swere in Portuguese: I) Análise do Comportamento, II) Behaviorismo Radical, III) B.F. Skinner, IV) EducaçãoInfantil, V) Ensino Fundamental, VI) Ensino Médio, VII) Ensino Superior, VIII) Educação de Jovens e Adultos, IX) EducaçãoProfissional e Tecnológica, X) EducaçãoIndígena, XI) Educação Especial, XII) Educação do Campo e XIII) EducaçãoAmbiental. The key words were divided into two groups: a) Behavior Analysis (I, II and III), and b) Education's levels and types (from IV to XIII). Each word of the first group was combined to each word of the second group. 35 workswere the outcome of these searches (15 doctoral thesis and 20 master dissertations).77,14\% of these works are from the southeast region of Brazil. 51,43\% of the selected works were from PUC-SP and UFSCar universities. In relation to pedagogic practices, matching-to-sample was the most frequent concept, it appeared in 18works.34,29\% of the selected works targeted elementary education population. The only type of education cited in the 35 works was special education.
\end{abstract}

Key-words: Behaviorism. Behavior Analysis. Education

\section{Inserción del análisis del comportamiento en la educación: el estado del conocimiento de tesis y disertaciones producidas entre 2010 y 2015}

Resumen: En el contexto educacional formal, el análisis del comportamiento estudia las relaciones que ocurren entre sujetos dentro de las aulas. La práctica pedagógica, o la enseñanza, se define como planificar situaciones que favorezcan el aprendizaje de los alumnos. El presente trabajo es una investigación bibliográfica del tipo estado del conocimiento. El objetivo es verificar en tesis y disertaciones producidas entre 2010 y 2015 como conceptos del Análisis del Comportamiento fueron aplicados en contextos educativos La recolección de datos fue hecha en el Portal Brasileño de Publicaciones Científicas en Acceso Abierto, a partir de las siguientes palabras clave: I) Análisis del comportamiento; II) Conductismo Radical; III) B. F. Skinner; IV) Educación infantil; V) Enseñanza Fundamental; VI) Enseñanza Media; VII) Enseñanza Superior; VIII) Educación de Jóvenes y Adultos; IX) Educación Profesional y Tecnológica; X) Educación Indígena; XI) Educación Especial; XII) Educación del Campo; y XIII) Educación Ambiental. Las palabras clave se dividieron en dos grupos: a) Análisis del comportamiento (I, II y III) y b) niveles y modalidades de enseñanza (de IV a XIII). Cada elemento del primer grupo se combinó con cada elemento del segundo grupo. Las búsquedas encontraron 35 trabajos (15 tesis y 20 disertaciones), de loscuales el 77,14\% procede de la región Sudeste. Pontificia Universidad 
Católica de São Paulo y Universidad Federal de São Carlos produjeron el 51,43\% de los trabajos seleccionados. Encuanto a las prácticas pedagógicas, el concepto más frecuente fue coincide con el sample, que apareció en 18 de éstos. Encuanto a los niveles de enseñanza, la enseñanza fundamental obtuvo la mayor concentración de trabajos: 34,29\%. Sobre las modalidades de enseñanza, sólo la Educación Especial fue citada.

Palabras clave: Conductismo. Análisis del comportamiento. Educación.

\section{Referências}

BANDURA, A. Social learning through imitation. In: JONES, M. R. (Ed.). Nebraska symposium on motivation. Lincoln: Univ. of Nebraska, 1962. p. 211-274.

BANDURA, A. Influence of model's reinforcement contingencies on the acquisition of imitative responses. Journal of Personality and Social Psychology, Washington, v. 1, n. 6, p. 589-585. jun. 1965.

BARROS, F. A. F. Os desequilíbrios regionais da produção técnicocientífica. São Paulo Perspectiva, São Paulo, v. 14, n. 3, p. 12-19, jul., 2000. Disponível em: <http://www.scielo.br/scielo.php?script=sci_ arttextEpid $=$ S0102-88392000000300004Elng $=$ enE $\mathrm{nrm}=$ iso $>$. Acesso em: 03 fev. 2017.

CARVALHO, C. S. N. Curso de especialização lato sensu: aprendizagem significativa, autonomia e práticas pedagógicas. 2015. 92 f. Dissertação (Mestrado Interdisciplinar em Educação, Linguagens e Tecnologias) Instituto de Educação, Universidade Estadual de Goiás, Goiânia, 2015.

CATANIA, A. C. Aprendizagem: comportamento, linguagem e cognição. Porto Alegre: Artmed, 1999.

GIOIA, P. S. A abordagem behaviorista radical transmitida pelo livro de psicologia direcionado à formação de professores. 2001. Tese (Doutorado em Psicologia da Educação) - Instituto de Psicologia, Pontifícia Universidade Católica de São Paulo, São Paulo, 2001.

HENKLAIN, M. H. O; CARMO, J. S. Contribuições da análise do comportamento à educação: um convite ao diálogo. Caderno de pesquisa, São Paulo, v. 43, n. 149, p. 704-723, ago., 2013. Disponível em: <http://www.scielo.br/scielo.php?script $=$ sci_arttext\& pid $=$ S0100$15742013000200016 \mathcal{E} 1 \mathrm{ng}=$ enE $\mathrm{nrm}=$ iso $>$. Acesso em: 10 out. 2016.

LOBATO, D. M. A educação profissional por competências: a prática pedagógica da modalidade aprendizagem do SENAI - Pará. 2011. 85 f. Dissertação (Mestrado em Educação) - Instituto de Educação, Universidade Federal do Pará, Belém, 2011.

MOROZ, M. Educação e autonomia: relação presente na visão de B. F. Skinner. Temas em Psicologia, Ribeirão Preto, v. 1, n. 2, p. 31-40, ago. 1993.

PACCA, F. C. Investigação de conhecimento teórico: aceitação/rejeição ao behaviorismo radical entre professores. Dissertação (Mestrado em 
Educação) - Programa de Pós-Graduação em Educação, Universidade Estadual Paulista "Júlio de Mesquita Filho", Marília, 2013.

SAVIANI, D. As concepções pedagógicas na história da educação brasileira. Campinas, 2005. Disponível em: <http://www.histedbr.fae.unicamp.br/ navegando/artigos_frames/artigo_036.html>. Acesso em 22 out. 2016.

SÉRIO, T. M. A. P. et al. Controle de estímulos e comportamento operante: uma (nova) introdução. 3. ed. São Paulo: SP. Educ, 2010.

SKINNER, B. F. Sobre o behaviorismo. 10. ed. São Paulo: Cultrix, 2006

SKINNER, B. F. Seleção por Consequências. Revista Brasileira de Terapia Comportamental e Cognitiva, São Paulo, v. 9, n. 1, p. 129-137, jan. 2007.

SKINNER, B. F. Ciência e Comportamento Humano. Brasília, DF:

Ed. UnB: FUNBEC, 2003.

SKINNER, B. F. Tecnologia do ensino. Tradução de Rodolpho Azzi.

São Paulo: EPU, 1972.

SKINNER, B. F. Teorias de aprendizagem são necessárias? Revista Brasileira de Análise do Comportamento, Belém, v. 1, n. 1, p. 105-124, maio, 2005.

TOURINHO, E. Z. Estudos conceituais na análise do comportamento. Temas em Psicologia da SBP, Ribeirão Preto, v. 7, n. 3, p. 213-222. 1999.

Submetido em: 23/07/2017. Aceito em: 19/12/2017. 